\title{
Primitive Juggling Sequences
}

\author{
Fan Chung Ron Graham
}

\section{Introduction}

Over the past two decades, a number of ways have been developed for representing periodic juggling patterns by finite sequences of nonnegative integers. In the most common of these, the entries of the sequence indicate how long the thrown object stays in the air. These juggling sequences have many interesting mathematical properties. In particular, each juggling sequence has associated with it a binary vector called its state. In this note, we analyze the juggling sequences associated with an arbitrary state, and show how to enumerate the number of such sequences which have a given length.

In the juggling vernacular, a juggling sequence (often called a siteswap by jugglers) is a sequence $T=\left(t_{1}, t_{2}, \ldots, t_{n}\right)$ of nonnegative integers satisfying the following modular condition [5]:

The quantities $i+t_{i}(\bmod n)$ are all distinct for $1 \leq i \leq n$.

The interpretation is this. Each $t_{i}$ denotes the amount of time that the ball (or other object) is in the air. A ball thrown by an amount $t_{i}$ at time $i$ means that that this ball will come down at time $i+t_{i}$. Often we will refer to $t_{i}$ as the height the ball is thrown. The preceding modular condition is just the constraint that juggling sequence is repeated indefinitely, then two balls don't come down at the same time. By convention, $t_{i}=0$ means that no ball is thrown at time $i$.

The number $n$ is called the period of $T$, and the quantity $\frac{1}{n} \sum_{i=1}^{n} t_{i}$ is called the average of $T$. For jugglers, the average of $T$ is the number of balls being juggled, and we will usually refer to it this way in what follows. Ordinarily, the sequence $T$ is repeated indefinitely. An example of a juggling sequence is $T_{0}=(5,3,0,4,2,5,2,6,1,2)$. Here, $T_{0}$ has period 10 and the number of balls is 3. It is an immediate consequence of the definition of a juggling sequence that the "number of balls" of a juggling sequence is always an integer (fortunately!). It is known [5], for example, that the number of juggling sequences with period $n$ and at most $b$ balls is exactly $(b+1)^{n}$. One can observe that it is possible to decompose $T_{0}=(5,3,0,4,2,5,2,6,1,2)$ into 3 shorter juggling sequences all with the same number of balls as follows: $(5,3,0,4)(2,5,2)(6,1,2)$. 
Furthermore, none of these shorter sequences can be further decomposed in this manner (and in fact, this decomposition is unique). It will turn out that these shorter juggling sequences are all examples of primitive juggling sequences. In the following sections, we expand on this concept, introducing the concept of $\sigma$-juggling sequences, and primitive $\sigma$-juggling sequences, where $\sigma$ denotes the "state" of the sequence (defined in the next section). We also show how to enumerate $\sigma$-juggling sequences and primitive $\sigma$-juggling sequences with period $n$.

As we will see, one use of primitive juggling sequences is that we can make new juggling sequences by taking arbitrary products of $\sigma$-primitive juggling sequences. For example, with the primitive juggling sequences $(3),(4,2),(5,3,1)$, $(4,5,3,0),(5,2,2)$, we can form the following new and complicated-looking juggling sequences (which are hard to perform in part because they are hard to remember!):

3334253134530522
42334233522453042423
35314242333453045305313531

\section{Preliminaries}

In the study of juggling sequences, a juggling state or landing schedule, denoted by $\sigma$, is a binary sequence $\left\langle\sigma_{1}, \sigma_{2}, \ldots, \sigma_{h}, \ldots\right\rangle$ that indicates the times at which the balls that are in the air at some particular time will land. To define the juggling state immediately after a particular throw has been made, let us take the time of this throw to be the time 0 . Then $\sigma_{i}=1$ if there is a ball in the air that will land at time $i$, and otherwise $\sigma_{i}=0$. Every $\sigma$ is actually an infinite binary sequence in which there are only finitely many nonzero entries. Ordinarily, we only write an initial part of $\sigma$ containing its nonzero entries, say $\sigma=\left\langle\sigma_{1}, \sigma_{2}, \ldots, \sigma_{h}\right\rangle$, with the understanding that $\sigma_{k}=0$ for $k>h$.

We now define the state $\sigma$ of a juggling sequence $T=\left(t_{1}, t_{2}, \ldots, t_{n}\right)$ to be the state immediately after the last throw $t_{n}$, where we assume that the sequence $T$ has been repeated infinitely many times in the past. Thus, we have $\sigma_{i}=1$ if and only if there is some $j \in\{1,2, \ldots, n\}$ and some $d>0$ such that $j+t_{j}=i+d n$. For example, the state of the juggling sequence $(4,1,5,2)$ is $\langle 1,1,0,1\rangle$ (see Figure 1). The number of 1's in $\sigma$, denoted by $|\sigma|$, is the number of balls in the pattern.

The act of juggling generates a sequence of juggling states which follows one rule: At a state $\sigma=\left\langle\sigma_{1}, \sigma_{2}, \ldots, \sigma_{h}\right\rangle$, if $\sigma_{1}=1$, the options for the next throw are a $j$-throw (or a throw of height $j$ ) for those $j$ 's with $\sigma_{j+1}=0$. If $\sigma_{1}=0$ then no ball is thrown, and this is a no-throw. So, after a $j$-throw, $\sigma$ is transformed into $\sigma^{\prime}$ where $\sigma_{j}^{\prime}=1$ and $\sigma_{k}^{\prime}=\sigma_{k+1}$ for $k \neq j$. After a no-throw, the new state 


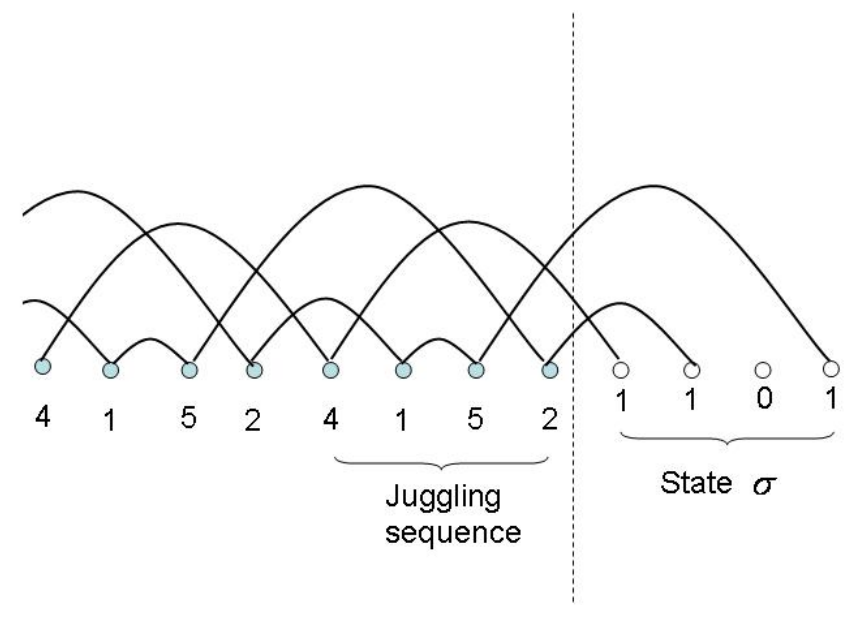

Figure 1: A juggling sequence and its associated state.

$\sigma^{\prime}$ satisfies $\sigma_{k}^{\prime}=\sigma_{k+1}$, the only option for all $k$. The number of balls always remains constant.

For a fixed $b$, the state diagram $D_{b}$ for $b$ balls is a directed graph with vertices being the juggling states $\sigma$ with $|\sigma|=b$, and with directed edges defined by the above transformations. In addition, the edge associated with a $t_{i}$-throw is labelled by $t_{i}$. Of course, $D_{b}$ is an infinite directed graph, and all the outdegrees of vertices $\sigma$ with $\sigma_{1}>0$ have infinite degree. For each $h>0$, we define the finite subgraph $D_{b, h}$ of $D_{b}$ to be the subgraph induced by those vertices $\sigma$ with $\sigma_{k}=0$ for $k>h$. (From a historical perspective, the notion of the state vector and the state diagram for juggling sequences appears to be due to Jack Boyce (see [10]). In Figure 2, the state diagram for 3 balls and height at most 5 is illustrated.

It is easy to see that a juggling sequence corresponds to a cycle in the state diagram (see [12], [16]). A prime juggling sequence is defined to be a juggling sequence with no repeated states. In other words, a prime juggling sequence is associated with a simple cycle in the state diagram. For example, the juggling sequence $(5,0,5,3,0,5,5,1)$ is a prime juggling sequence with state $\sigma=\langle 1,0,1,1,0\rangle$ as can be seen in the state diagram shown in Figure 2 .

Note that while $(4,2)$ and $(5,0,4)$ are both juggling sequences with 3 balls, the concatenation (or "product") $(4,2)(5,0,4)=(4,2,5,0,4)$ is not a juggling sequence. In this sense, we cannot take arbitrary products of prime juggling sequences to get new juggling sequences. The reason for this is basically because these two juggling sequences have different states. We will elaborate on this more in the next section. 


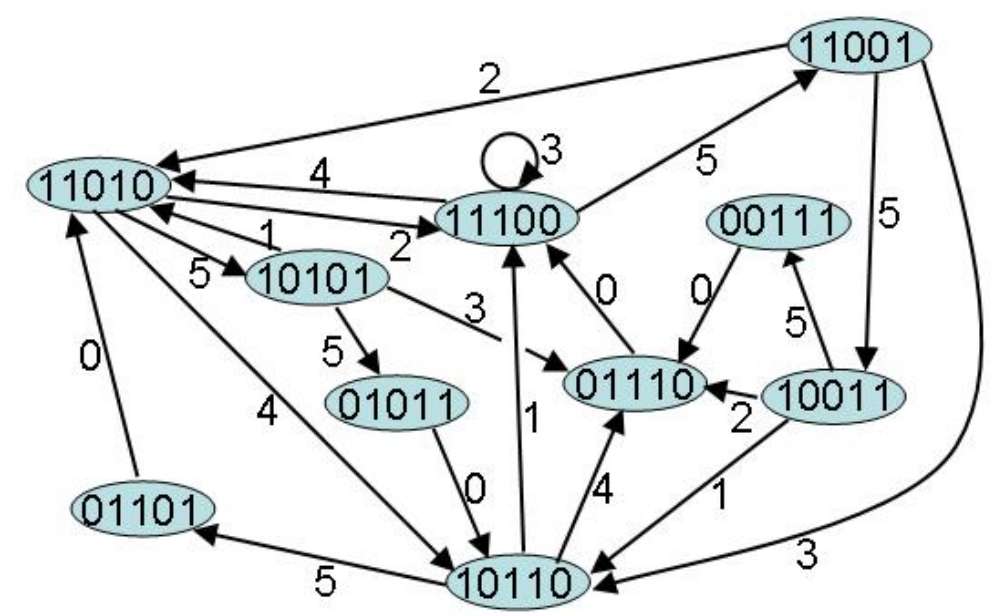

Figure 2: The state diagram for 3 balls and height 5 .

It is clear from the definition of the state diagram $D_{b}$ that the product of any two juggling sequences with the same state is also a juggling sequence with that state. In the juggling literature, there is a distinguished state $\sigma^{(b)}$, called the ground state for $b$ ball patterns, defined by $\sigma^{(b)}=\langle 1,1, \ldots, 1\rangle$, where there are $b$ 1 's. Examples of juggling sequences which have this ground state are $(3),(4,2)$, $(5,3,1),(5,5,5,0,0)$, etc., in $D_{3}$. In general, a juggling sequence $T$ with state $\sigma$ (i.e., which corresponds to a cycle in $D_{b}$ starting and ending at $\sigma$ ) will be called a $\sigma$-juggling sequence.

Let us define a $\sigma$-juggling sequence to be primitive if it cannot be written as the product of two shorter $\sigma$-juggling sequences. Thus, a $\sigma$-juggling sequence is primitive if the corresponding cycle in the state diagram does not visit the state $\sigma$ more than once. With this definition, prime juggling sequences are primitive, but not conversely. For example, in $D_{3},(2,3,5,2)$ is a primitive $\langle 1,1,0,1,0\rangle$ juggling sequence that is not prime. We might point out that while $(2,3,5,2)$ is a primitive $\langle 1,1,0,1,0\rangle$-juggling sequence, the related juggling sequence $(3,5,2,2)$ is not a primitive $\langle 1,1,1,0,0\rangle$-juggling sequence. That is, while in juggling we would not distinguish between these two, the analysis does break them into two classes. The main question we address in this note is to count the number of primitive $\sigma$-juggling sequences which have period $n$. 


\section{$3 \quad$ Enumerating ground state juggling sequences}

As a warm-up to the general case treated in the next section, we will first consider the simplest case, which is for juggling sequences in the ground state, i.e., with state $\sigma=\sigma^{(b)}=\langle 1,1, \ldots, 1\rangle$ (where there are $b 1$ 's). Let us denote by $J(b, n)=J\left(\sigma^{(b)}, n\right)$ the number of juggling sequences with state $\sigma^{(b)}$ and period $n$. Also, let $P(b, n)=P\left(\sigma^{(b)}, n\right)$ denote the number of primitive $\sigma^{(b)}$-juggling sequences with period $n$.

Although there is an extensive literature on juggling (as well as a great deal of interest by many jugglers), relatively few of these numbers have been computed, let alone analyzed. It turns out that these numbers have many interesting properties and are related to some known sequences in the literature. We begin with a warm-up theorem.

Theorem 1 For any $b$ and $n$, the number $J(b, n)$ satisfies

$$
J(b, n)= \begin{cases}(b+1)^{n-b} b ! & \text { if } n \geq b, \\ n ! & \text { otherwise. }\end{cases}
$$

Proof: First, observe that the $\sigma$-juggling sequences of period $n$ are precisely the sequences $T=\left(t_{1}, t_{2}, \ldots, t_{n}\right)$ with the property that $\left\{t_{i}+i: i \in[1, n]\right\}=$ $[b+1, b+n]$. For, we can't have $t_{i}+i<b+1$ since this would imply that two balls would land at the same time, and we can't have $t_{i}+i>b+n$, since in this case we wouldn't return to state $\sigma^{(b)}$ after $n$ steps. Thus, for every $i, b+1 \leq t_{i}+i \leq b+n$. The modular condition now implies that $\left\{t_{i}+i: i \in[1, n]\right\}=[b+1, b+n]$. Consequently, the problem of determining the number of $\sigma^{(b)}$-juggling sequences of period $n$ is the same as determining the number of permutations $\pi$ on $\{1, \ldots, n\}$ such that $\pi(i) \geq b+i$, since each such permutation corresponds to a juggling sequence $T$ with $t_{i}=\pi(i)+b-i$.

We consider the matrix $M$ with columns and rows indexed by $\{1,2, \ldots, n\}$. The entries $M(i, j)$ of $M$ are 1 if $j \geq i-b$, and 0 otherwise. It is not hard to see that $J(b, n)=\operatorname{Per}(M)$, the permanent of $M$. Recall that the permanent of a square matrix is similar to the determinant of the matrix except no minus signs are used when summing the terms. It is straightforward to compute that if $n \geq b$ then $\operatorname{Per}(M)=(b+1)^{n-b} b$ !, as desired. Finally, if $n<b$ then the above argument shows that $J(n, b)=n$ !, and the proof is complete.

We can now derive the number $P(b, n)$ of primitive juggling sequences from $J(b, n)$ using the following recurrence relation:

$$
J(b, n)=P(b, n)+P(b, n-1) J(b, 1)+\cdots+P(b, 1) J(b, n-1)
$$

with $P(b, 1)=J(b, 1)=1$. 
For a fixed $b$, consider the generating function $f_{b}(x)$ defined by

$$
f_{b}(x)=1+\sum_{i=1}^{\infty} J(b, i) x^{i} .
$$

Also define

$$
g_{b}(x)=\sum_{i=1}^{\infty} P(b, i) x^{i} .
$$

¿From (1), we have

$$
f_{b}(x)-1=g_{b}(x) f_{b}(x) .
$$

Therefore, $g_{b}(x)$ satisfies

$$
g_{b}(x)=1-\frac{1}{f_{b}(x)} .
$$

For example, for the case of $b=2$ (using the above values of $J(2, n)$ ) we have

$$
\begin{aligned}
f_{2}(x) & =1+x+\sum_{k=2}^{\infty} 2 \cdot 3^{k-2} x^{k} \\
& =\frac{1-2 x-x^{2}}{1-3 x} \\
& =1+x+2 x^{2}+6 x^{3}+18 x^{4}+54 x^{5}+162 x^{6}+486 x^{7}+\cdots, \\
g_{2}(x) & =\frac{x-x^{2}}{1-2 x-x^{2}} \\
& =x+x^{2}+3 x^{3}+7 x^{4}+17 x^{5}+41 x^{6}+99 x^{7}+\cdots .
\end{aligned}
$$

A quick check in Sloane [13] shows that the above sequence of coefficients of $g_{b}(x)$ (which are the values of $P(n, 2)$ ) and its generating function are well known. Among their many interpretations are the following:

(i) The numbers $P(n, 2)$ are numerators of the continued fraction convergents to $\sqrt{2}$.

(ii) $P(n, 2)$ is the number of $n$-step non-self-intersecting paths starting at $(0,0)$ with steps of types $(1,0),(-1,0)$ or $(0,1)$ (see $[15])$.

(iii) $P(2 k, 2)=\mathcal{T}(k, 3)$ and $P(2 k+1,2)=\mathcal{S}(2 k, 2 \sqrt{2})$ where $T(k, x)$ and $S(k, x)$ are Chebyshev polynomials of the first and second kinds, respectively.

The connection to primitive juggling sequences of 2 balls seems to be new. 
For the case of $b=3$, we have

$$
\begin{aligned}
f_{3}(x) & =1+x+2 x^{2}+\sum_{k=3}^{\infty} 2 \cdot 3 \cdot 4^{k-3} x^{k} \\
& =\frac{1-3 x-2 x^{2}-2 x^{3}}{1-4 x} \\
& =1+x+2 x^{2}+6 x^{3}+24 x^{4}+96 x^{5}+384 x^{6}+\cdots, \\
g_{3}(x) & =1-\frac{1-4 x}{(1-4 x)\left(1+x+2 x^{2}\right)+6 x^{3}} \\
& =\frac{x-2 x^{2}-2 x^{3}}{1-3 x-2 x^{2}-2 x^{3}} \\
& =x+x^{2}+3 x^{3}+13 x^{4}+47 x^{5}+173 x^{6}+639 x^{7}+\cdots .
\end{aligned}
$$

The sequence for $P(3, n)$ above is listed in Sloane [13] as sequence number A084519, the first 25 terms of which were computed by Antti Karttunen. It was conjectured by Benoit Cloitre that they satisfy the following recurrence relation:

$$
P(3, n)=3 P(3, n-1)+2 P(3, n-2)+2 P(3, n-3) .
$$

This in fact now follows easily from the above explicit generating function in (2).

For general $b$, the generating function $g_{b}(x)$ is

$$
g_{b}(x)=1-\frac{1-(b+1) x}{1-x \sum_{k=0}^{b-1}(b-k) k ! x^{k}}
$$

which is obtained by using the expression for $f_{b}(x)$ :

$$
f_{b}(x)=\frac{1-x \sum_{k=0}^{b-1}(b-k) k ! x^{k}}{1-(b+1) x} .
$$

Holding $n$ fixed and letting $b$ tend to infinity, the generating function $g_{b}(x)$ stabilizes to the following well known function:

$$
g(x)=1-\frac{1}{\sum_{k=0}^{\infty} k ! x^{k}}=x+x^{2}+3 x^{3}+13 x^{4}+71 x^{5}+461 x^{6}+\cdots .
$$

This follows from the observation that $J(b, n)=n$ ! for $b \geq n-1$, and the fact that $P(b, n)$ is determined by $J(b, 1), \ldots, J(b, n)$. For example, the coefficient of $x^{k}$ in $g(x)$ can be interpreted as (see [13] \# A003319):

(i) the number of (indecomposable) permutations of $[1, k]$ not fixing $[1, j]$, for $0<j<k$ (see [8]),

(ii) the maximal number of subgroups of index $k-1$ in any 2-generator group (see [15]), 
(iii) the dimension of the homogeneous components of the space of primitive elements of the Malvenuto-Reutenauer Hopf algebra of permutations (see $[1,11])$.

For general $b$, the recurrence relation for $P(b, n)$ can be derived from the generating function $g_{b}(x)$ in (4):

$$
P(b, n)=\sum_{k=0}^{b-1}(b-k) k ! P(b, n-k-1)
$$

which explains the coefficients in (3).

\section{Enumerating general $\sigma$-juggling sequences}

In this section we consider $\sigma$-juggling sequences for general state vectors $\sigma$.

Lemma 1 A necessary condition for a $\sigma$-juggling sequence of period $n$ to exist is that for all $k>n, \sigma_{k}=1$ implies $\sigma_{k-n}=1$.

Proof: First, observe that if we are in state $\sigma$ and we execute the sequence of throws $t_{1}, t_{2}, \ldots, t_{m}$ then the new state will be $\sigma^{\prime}$, where $\sigma_{j}^{\prime}=1$ if and only if either $\sigma_{j+m}^{\prime}=1$, or for some $i, t_{i}+i=j+m$. In other words, a ball will land in $j$ beats if and only if either there was already a ball in the air that was scheduled to land then, or one of the $m$ balls that were thrown will land then. If $T$ is a $\sigma$-juggling sequence with period $n$, then after $n$ steps we must return to the state $\sigma$. Therefore, $\sigma_{j}=1$ if and only if either $\sigma_{j+n}=1$, or for some $i$, $t_{i}+i=j+n$. So in particular, if $\sigma_{j+n}=1$ then $\sigma_{j}=1$.

We note that the condition in the preceding lemma is equivalent to the requirement that for all $k, \sigma_{k}=0$ implies $\sigma_{k+n}=0$.

For each value of $n$ satisfying the conditions of the preceding lemma, we define a set $U_{\sigma, n}=\left\{u_{1}, u_{2}, \ldots, u_{n}\right\}$ as follows. For $1 \leq i \leq n, u_{i}$ is defined to be the least value $i+d n$ for $d \geq 0$ such that $\sigma_{i+d n}=0$, where, as above, we assume that $\sigma_{j}=0$ for $j>h$.

Next, for these $n$, we define the matrix $M_{\sigma, n}$ as follows. The rows and columns of $M_{\sigma, n}$ are indexed by $\{1,2, \ldots, n\}$. We define $M_{\sigma, n}(i, j)$ to be 1 if $i \leq u_{j}$, and 0 otherwise. Therefore in the $j$ th column, there are exactly $\min \left\{u_{j}, n\right\} 1$ 's above $\max \left\{n-u_{j}, 0\right\} 0$ 's.

Let $J(\sigma, n)$ denote the number of $\sigma$-juggling sequences, and let $P(\sigma, n)$ denote the number of primitive $\sigma$-juggling sequences. 
Theorem 2 For a given state vector $\sigma$ and period $n$ satisfying Lemma 1, the number $J(\sigma, n)$ of $\sigma$-juggling sequences satisfies

$$
J(\sigma, n)=\operatorname{Per}\left(M_{\sigma, n}\right) .
$$

Proof: Observe that the modular constraints on the $t_{i}$ imply that for each $j$ in $[1, n]$, there must be a unique $i$ such that $t_{i}+i=j+n d$ for some $d$. By Lemma $1, \sigma_{j+n d}=1$ if $j+n d<u_{j}$ and $\sigma_{j+n d}=0$ if $j+n d \geq u_{j}$. Thus, we cannot have $t_{i}+i=j+n d<u_{j}$, because then we would have two balls landing at the same time. On the other hand, we cannot have $t_{i}+i=j+n d>u_{j}$ since in this case we wouldn't return to state $\sigma$ after $n$ steps. (Note the similarity to the argument in Theorem 1.) We conclude from this that $\left\{t_{i}+i: i \in[1, n]\right\}$ must be equal to $U_{\sigma, n}$.

For each permutation $\pi$ of $\{1,2, \ldots, n\}$ which contributes 1 to $\operatorname{Per}\left(M_{\sigma, n}\right)$, we can define $T=\left(t_{1}, t_{2}, \ldots, t_{n}\right)$ by setting $t_{i}=u_{\pi(i)}-i$. From the definition of $M(\sigma)$, we see that $t_{i} \geq 0, t_{i}+i$ are distinct modulo $n$, and, in fact, $T$ is a $\sigma$-juggling sequence with period $n$. Furthermore, for any $\sigma$-juggling sequence $T=\left(t_{1}, t_{2}, \ldots, t_{n}\right)$, we can define a permutation $\pi$ with $\pi(i) \equiv t_{i}+i(\bmod n)$ which contributes 1 to the permanent. This proves the theorem.

We next state a lemma useful for evaluating the permanent in the preceding theorem. Of course, the value of the permanent of a matrix is unchanged by permutating the rows and columns of the matrix.

Lemma 2 Suppose $M$ is a binary matrix with rows and columns indexed by $\{1,2, \ldots, n\}$ such that the $j$ th column of $M$ consists of $v_{j} 1$ 's above $n-v_{j} 0$ 's. Furthermore, assume that $0 \leq v_{1} \leq v_{2} \leq \cdots \leq v_{n} \leq n$. Then

$$
\operatorname{Per}(M)=\prod_{j=1}^{n}\left(v_{j}-j+1\right) .
$$

Proof: The proof follows by an easy induction on $n$.

The recurrence relation for $P(\sigma, n)$ is quite similar to that in (1), namely,

$$
J(\sigma, n)=P(\sigma, n)+P(\sigma, n-1) J(\sigma, 1)+\cdots+P(\sigma, 1) J(\sigma, n-1)
$$

except that we have $P(\sigma, 1)=0=J(\sigma, 1)$ if $\sigma$ is not the ground state.

We can define the generating functions

$$
f_{\sigma}(x)=1+\sum_{i=1}^{\infty} J(\sigma, i) x^{i}
$$

and

$$
g_{\sigma}(x)=\sum_{i=1}^{\infty} P(\sigma, i) x^{i}
$$


and again we have

$$
g_{\sigma}(x)=1-\frac{1}{f_{\sigma}(x)}
$$

¿From this representation, we can obtain various kinds of specific information about $P_{\sigma, n}$.

As an example, let $\sigma=\langle 1,0,1,1,1,0,1,1\rangle$. It can then be checked that the only $n$ for which $\sigma$-juggling sequences with period $n$ exist are $n=4$ and $n \geq 7$. For these values of $n$ we have $U_{\sigma, 4}=\{2,9,11,12\}, U_{\sigma, 7}=\{2,6,10,11,12,14,15\}$, $U_{\sigma, 8}=\{2,6,9,11,12,13,15,16\}$, and in general, for $n \geq 8$,

$$
U_{\sigma, n}=\{2,6,9,10, \ldots, n, n+1, n+3, n+4, n+5, n+7, n+8\} .
$$

This implies (by Lemma 1) that:

$$
\begin{aligned}
f_{\sigma}(x) & =1+12 x^{4}+2 \cdot 5 \cdot 5 ! x^{7}+2 \cdot 5 \cdot 6 ! x^{8}+\cdots+2 \cdot 5 \cdot 7^{n-8} \cdot 6 ! x^{n}+\cdots \\
& =1+12 x^{4}+1200 x^{7}+\frac{7200 x^{8}}{1-7 x} \\
& =\frac{1-7 x+12 x^{4}-84 x^{5}+1200 x^{7}-1200 x^{8}}{1-7 x} \\
& =1+12 x^{4}+1200 x^{7}+7200 x^{8}+50400 x^{9}+352800 x^{10}+2469600 x^{11} \cdots
\end{aligned}
$$

and

$$
\begin{aligned}
g_{\sigma}(x) & =\frac{12 x^{4}\left(1-7 x+100 x^{3}-100 x^{4}\right)}{1-7 x+12 x^{4}-84 x^{5}+1200 x^{7}-1200 x^{8}} \\
& =12 x^{4}+1200 x^{7}+7056 x^{8}+50400 x^{9}+352800 x^{10}+2440800 x^{11}+\cdots .
\end{aligned}
$$

Thus, the values of $P(n)=P(\sigma, n)$ satisfy the linear recurrence

$P(n)=7 P(n-1)-12 P(n-4)+84 P(n-5)-1200 P(n-7)+1200 P(n-8)$,

which implies that $P(\sigma, n)$ grows like $c \cdot \rho^{n}$, where $\rho=6.991237188 \ldots$ is the largest real root of $x^{8}-7 x^{7}+12 x^{4}-84 x^{3}+1200 x-1200$.

\section{Concluding remarks}

We close with a few unanswered questions which we feel are worth pursuing.

- It seems intuitively clear that among all $b$ ball state vectors $\sigma$, for each $n$ $P(\sigma, n)$ is largest when $\sigma$ is the ground state $\sigma^{(b)}$. Is this true?

- Are there many other sequences of $J(\sigma, n)$ and $P(\sigma, n)$ for various state vectors $\sigma$ which have arisen in other contexts? We haven't done any real exploration of this question but the fact that we found the three mentioned 
in the earlier sections makes us believe that there probably are. Here are a few others:

$$
g_{\langle 0,1,1\rangle}(x)=2 x^{3}\left(1+3 x+9 x^{2}+25 x^{3}+69 x^{4}+189 x^{5}+517 x^{6}+\cdots\right)
$$

whose coefficients form Sequence A077846 in [13],

$$
g_{\langle 0,0,1\rangle}(x)=x^{3}\left(1+2 x+4 x^{2}+7 x^{3}+12 x^{4}+20 x^{5}+\cdots\right)
$$

whose coefficients are Fibonacci numbers minus 1 (Sequence A000071 in [13]), and

$$
g_{\langle 0,1\rangle}(x)=x\left(x+2 x^{2}+3 x^{3}+4 x^{4}+5 x^{5}+6 x^{6}+\cdots\right)
$$

which is Sequence A000027 in [13]. Of course, a good check as to whether a sequence has appeared in the literature before is to consult Sloane's Online Encyclopedia of Integer Sequences [13]. Here we list several generating functions of primitive juggling sequences that have not yet been identified at the time this paper was written:

$$
\begin{aligned}
g_{\langle 1,0,1\rangle}(x) & =\frac{2 x^{2}-2 x^{3}}{1-3 x+2 x^{2}-2 x^{3}} \\
& =2 x^{2}+4 x^{3}+8 x^{4}+20 x^{5}+52 x^{6}+132 x^{7}+\cdots, \\
g_{\langle 0,1,1,1\rangle}(x) & =\frac{x^{4}-2 x^{5}-2 x^{6}}{1-4 x+x^{4}-2 x^{5}-2 x^{6}} \\
& =x^{4}+2 x^{5}+6 x^{6}+24 x^{7}+95 x^{8}+380 x^{9}+\cdots .
\end{aligned}
$$

As mentioned before, even for ground states $\sigma^{(b)}, b \geq 4, g_{\sigma^{(b)}}(x)$ is not fully understood.

- Can the prime juggling sequences with state $\sigma$ and period $n$ be enumerated along the same lines that primitive juggling sequences were? This appears at present to be a more difficult problem.

- Can the primitive juggling sequences with state $\sigma$, period $n$, and height at most $h$ be effectively enumerated? This means that every $t_{i}$ is at most $h$ for the allowable juggling sequences $T$. More generally, one could require that $h_{1} \leq t_{i} \leq h_{2}$ for all $i$.

- What are the analogous results for multiplex juggling patterns? In this case, many balls (i.e., more than one) are allowed to be thrown and caught at each time instant. There have been a number of interesting papers published dealing with other aspects of multiplex juggling sequences (see [7], [12], [14]). 


\section{Acknowledgements}

The authors wish to acknowledge the insightful comments that Adam Chalcraft, Steve Butler and the referees contributed during the writing of this note. The research of Fan Chung was supported in part by NSF Grants DMS 0457215, ITR 0205061 and ITR 0426858. The research of Ron Graham was supported in part by NSF Grant CCR 0310991.

\section{References}

[1] M. Aguiar and F. Sottile, Structure of the Malvenuto-Reutenauer Hopf algebra of permutations, Adv. Math., 191, (2005), 225-275.

[2] J. Buhler and R. Graham, Fountains, showers, and cascades, The Sciences, (January-February 1984), 44-51.

[3] J. Buhler and R. Graham, A note on the binomial drop polynomial of a poset, J. Combin. Theory Ser. A, 66, (1994), 321-326.

[4] J. Buhler and R. Graham, Juggling patterns, passing, and posets, Mathematical Adventures for Students and Amateurs, MAA Publications, (2004), 99-116.

[5] J. Buhler, D. Eisenbud, R. Graham and C. Wright, Juggling drops and descents, Amer. Math. Monthly 101, (1994), 507-519.

[6] F. Chung and R. Graham, Universal juggling cycles, preprint.

[7] R. Ehrenborg and M. Readdy, Juggling and applications to $q$-analogues, Discrete Math. 157, (1996), 107-125.

[8] I. M. Gessel and R. P. Stanley, Algebraic enumerations, Handbook of Combinatorics, Vol. II, Elsevier, Amsterdam, (1995), 1021-1061.

[9] R. L. Graham, D. E. Knuth and O. Patashnik, Concrete Math. A Foundation for Computer Science, Addison-Wesley, Reading, MA, (1994).

[10] Juggling Information Service at www.juggling.org

[11] S. Poirier and C. Reutenauer, Algebres Hopf de tableaux, Ann Math. Quebec, 19, (1995), 79-90.

[12] B. Polster, The Mathematics of Juggling, Springer, New York, 2000.

[13] N. Sloane, Online Encyclopedia of Integer Sequences, http://www.research.att.com/ njas/sequences/.

[14] J. D. Stadler, Juggling and vector compositions, Discrete Math., 258, (2002), 179-191. 
[15] R. P. Stanley, Enumerative Combinatorics, Volume 1, (1986).

[16] G. S. Warrington, Juggling probabilities, American Mathematical Monthly, 112, No. 2, (2005), 105-118.

Fan Chung received her B.S. in Mathematics from Taiwan National University, and her Ph.D. in Mathematics from the University of Pennsylvania under the guidance of Herb Wilf. Since graduating, she has been a Member of the Technical Staff at Bell Laboratories, Division Manager of Mathematics Research at Bellcore, and the Class of 1963 Professor of Mathematics at the University of Pennsylvania. In addition, she has held visiting positions at Harvard University and the Institute for Advanced Study. She currently occupies the Akamai Chair of Internet Mathematics in the Mathematics and Computer Science Departments at the University of California at San Diego. Her current interests include spectral graph theory, quasi-randomness, random graphs and complex networks, water-color painting, and enjoying working with her favorite coauthor.

Ron Graham received his B.S. in Physics from the University of Alaska, and his Ph.D. in Mathematics from the University of California at Berkeley in the distant past. Much of his career was spent at Bell Laboratories and AT\&T Labs (which is not the same but only the cognoscenti know the difference). He has also enjoyed visiting (or part-time) positions at Caltech, Princeton, Rutgers, Stanford, and UCLA. He currently holds the Irwin and Joan Jacobs Chair of Computer and Information Science at the University of California at San Diego. His current interests include additive combinatorics, Ramsey theory, quasi-randomness, number theory, combinatorial geometry, theoretical computer science, and working with his favorite coauthor. In addition, he is probably the only person who is a former President of both the Mathematical Association of America and the International Jugglers Association. 